\title{
A recombination suppressor contributes to ecological speciation in OSTRINIA moths
}

\author{
CB Wadsworth, X Li and EB Dopman
}

Despite unparalleled access to species' genomes in our post-genomic age, we often lack adequate biological explanations for a major hallmark of the speciation process-genetic divergence. In the presence of gene flow, chromosomal rearrangements such as inversions are thought to promote divergence and facilitate speciation by suppressing recombination. Using a combination of genetic crosses, phenotyping of a trait underlying ecological isolation, and population genetic analysis of wild populations, we set out to determine whether evidence supports a role for recombination suppressors during speciation between the $Z$ and $E$ strains of European corn borer moth (Ostrinia nubilalis). Our results are consistent with the presence of an inversion that has contributed to accumulation of ecologically adaptive alleles and genetic differentiation across roughly $20 \%$ of the Ostrinia sex chromosome ( 4 Mb). Patterns in Ostrinia suggest that chromosomal divergence may involve two separate phases—one driving its transient origin through local adaptation and one determining its stable persistence through differential introgression. As the evolutionary rate of rearrangements in lepidopteran genomes appears to be one of the fastest among eukaryotes, structural mutations may have had a disproportionate role during adaptive divergence and speciation in Ostrinia and in other moths and butterflies.

Heredity (2015) 114, 593-600; doi:10.1038/hdy.2014.128; published online 28 January 2015

\section{INTRODUCTION}

A key feature of the speciation process is the evolving structure of genetic differentiation between populations that culminates in genetic divergence across much of the genomes of daughter taxa. Indeed, genomic patterns of evolutionary divergence across the speciation continuum promise to reveal not only the functional nature of adaptive evolution and reproductive isolation (Storz, 2005) but also the processes underlying speciation (Pinho and Hey, 2010). Consequently, a major research theme has emerged that aims to characterize genome-wide patterns of differentiation between diverging populations, races and species. Pockets of divergence or 'genomic islands of speciation,' as well as larger 'continents,' have been uncovered and could reflect the effect of genes contributing to reproductive isolation ('barrier loci') (Dopman et al., 2005; Turner et al., 2005; Feder, Egan et al., 2012). However, connecting these fundamental genetic signatures with their biological cause remains elusive (Noor and Bennett, 2009; Dopman, 2011; Nachman and Payseur, 2012; Cruickshank and Hahn, 2014).

Chromosomal rearrangements such as inversions are thought to facilitate speciation and promote the origin of evolutionary divergence by suppressing recombination. When speciation occurs in the face of gene flow, suppressed recombination in a heterokaryotypic hybrid offspring may cause the entire inverted segment to behave as a single linked unit (Noor et al., 2001; Rieseberg, 2001). Consequently, only a small number of barrier loci might be required to protect a large chromosomal band, possibly several megabases in length, from gene flow. Because of restricted gene flow, barrier and locally adaptive alleles are thought to accumulate within inversions leading to increasing divergence until speciation is complete (Navarro and Barton, 2003). Finally, adaptive alleles that are captured by inversions may rapidly drive their fixation, creating long-range hitch-hiking across the nonrecombining rearranged interval (Kirkpatrick and Barton, 2006; Kirkpatrick, 2010; Feder et al., 2011). In the absence of structural mutations, reduced recombination in collinear regions could also lead to evolutionary divergence; however, the footprint of genetic differentiation and opportunity for the accumulation of barrier loci might be limited (Butlin, 2005; West and Via, 2008; Feder et al., 2012). Thus, changes in recombination rate because of structural mutations might be the key to speciation with gene flow, and they may therefore help to explain many instances of genomic islands of speciation.

In this study, we evaluate the role of suppressed recombination for the evolution of a modestly sized genomic island of speciation between a pair of moth lineages. The ' $Z$ ' and 'E' strains of European corn borer (ECB, Ostrinia nubilalis) are incipient species that colonized North America $\sim 100$ ya without an appreciable bottleneck from allopatric locations in Italy (mixture of $\mathrm{E}$ and $\mathrm{Z}$ strains) and Hungary (exclusively Z-strain; Smith, 1920; Caffrey, 1927; Klun and Cooperators, 1975; Dopman, 2011). Strains are recently diverged ( 100 000 ya; Malausa et al., 2007) and are textbook examples of speciation (Coyne and Orr, 2004), in which one species is splitting into two through the evolution of many forms of reproductive isolation (Dopman et al., 2010). The sex (Z) chromosome harbors several known genetic factors for adaptation and/or barrier traits, including those underlying behavioral isolation (Roelofs et al., 1987; Dopman et al., 2004) and temporal isolation (Glover et al., 1992; Dopman et al., 2005). Current and historical gene exchange between 
ECB strains is likely, as indicated by hybridization in Europe and North America (Dopman et al., 2010; Coates et al., 2013), geographic variation in strength of reproductive isolation (ranging from 0.91 to 0.99; Dopman et al., 2010) and molecular evidence for an isolationwith-migration model of divergence ( $2 \mathrm{Nm} \sim 10$; Malausa et al., 2007). Whereas most loci show extensive shared polymorphism (for example, $F_{\mathrm{ST}}<0.05$ ), a possible island of speciation along an $\sim 1$-cM swath of the $\mathrm{Z}$ chromosome consists of four genes ( $T p i$ and three olfactory receptors, ORs) and reveals ECB strains as nearly reciprocally monophyletic (for example, $F_{\mathrm{ST}}>0.7$; Dopman et al., 2005; Dopman, 2011; Lassance et al., 2011). Evolutionary divergence can be explained by several tightly linked barrier loci on the sex chromosome or by a regional selective sweep at these or other loci. However, such interpretations must be viewed as incomplete without proper tests of suppressed recombination and its repercussions for the genomic zone of influence for adaptation and/or barrier loci. Indeed, a possible signature of an inversion between $\mathrm{Z}$ and $\mathrm{E}$ strains was detected on the $Z$ chromosome (Dopman et al., 2004), in which the lowest estimated recombination rate across the entire 1697-cM genetic linkage map was found in the vicinity of the Tpi/ORs loci (Figure 1).

If inversions are important during speciation and the evolution of genetic differentiation, at least three testable predictions have been made (Faria and Navarro, 2010). First, patterns of gene flow should be higher within collinear versus rearranged regions (Rieseberg, 2001; Feder et al., 2005; Kulathinal et al., 2009; McGaugh and Noor, 2012). Second, traits involved in reproductive isolation should map to regions located within chromosomal rearrangements (Noor et al., 2001; Feder et al., 2005; Lowry and Willis, 2010). Finally, genotyping hybrid offspring in the laboratory ought to reveal evidence for suppressed recombination (Feder et al., 2003; Kulathinal et al., 2009). By using a combination of genetic crosses, phenotyping of a trait contributing to reproductive isolation, and population genetic analysis, we set out to test these predictions for the ECB sex chromosome.

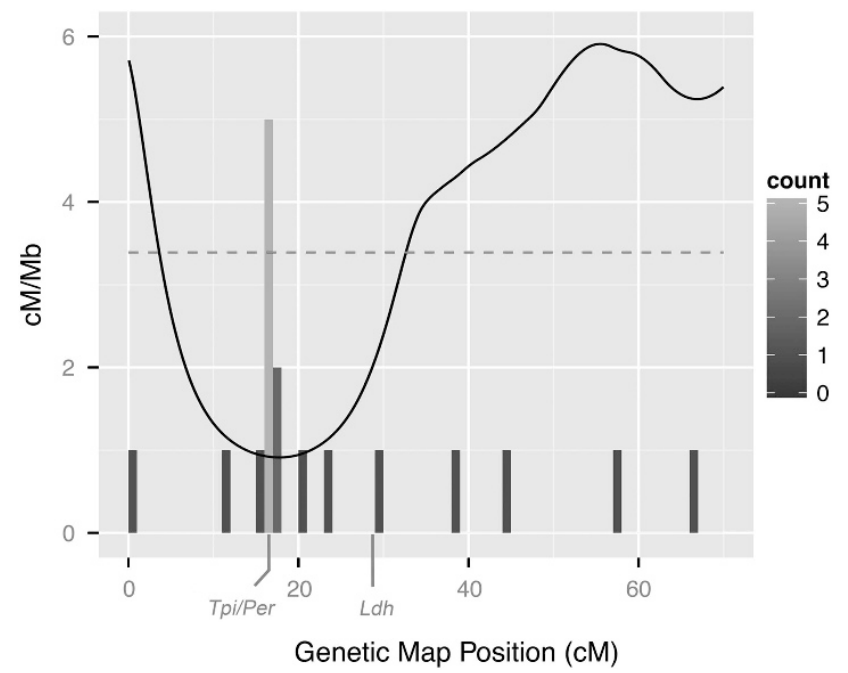

Figure 1 Local recombination rates (cM/Mb, black line) and marker densities (count) on the ECB sex $(Z)$ chromosome. The dashed red line indicates the genome-wide average. Genetically differentiated loci Tpi and ORs ( $\left.F_{\mathrm{ST}}>0.7\right)$ are located in the low-recombination region $(\sim 17 \mathrm{cM})$. Adapted from Dopman et al. (2004).

\section{MATERIALS AND METHODS}

\section{Recombination suppression}

To estimate variation in recombination frequencies surrounding the genetically differentiated region of the ECB sex chromosome, we generated three-point test crosses within and between $\mathrm{Z}$ and $\mathrm{E}$ strains. Crosses used insects collected as caterpillars, pupae and adults from the New York State, USA $(n \sim 500$ males and $n \sim 500$ females each from East Aurora, Geneva and Bouckville). Laboratory populations were maintained by mass rearing $\sim 100$ males and $\sim 100$ females each generation. The female sex-pheromone blend is strainspecific. Z-strain females produce a 3:97 ratio of E/Z11-14:OAc, whereas E-strain females use a 99:1 ratio. Strain identities of all parental stocks were confirmed by genotyping $P g F A R$, the autosomal locus that determines the female sex-pheromone blend (Lassance et al., 2010; Supplementary Table S1).

Recombination is restricted to males in many Lepidoptera (Dopman et al., 2004). Therefore, in backcross pedigrees only F1 males are informative. Here, between-strain backcrosses (referred to as ' $\mathrm{Z} \times \mathrm{E}$ ') involved a $\mathrm{Z}$ grandmother, an $E$ grandfather, an $F 1$ male and a recurrent $Z$-strain mother. Within-strain crosses (referred to as ' $\mathrm{Z} \times \mathrm{Z}$ ') had a similar design but were exclusively from the Z-strain.

The ECB diverged from Bombyx mori (the silk moth) 100 Mya (Pringle et al., 2007); however, the species pair shows a high degree of conservation in gene order like many other Lepidoptera (Pringle et al., 2007; d'Alencon et al., 2010; Kroemer et al., 2011). Therefore, we relied on the B. mori genome to identify evenly spaced sex-linked mapping loci for the ECB, whose genome is incomplete. Markers consisted of Tpi at $9 \mathrm{Mb}, P e r$ at $13 \mathrm{Mb}$ and $L d h$ at $17.4 \mathrm{Mb}$ (International Silkworm Genome Consortium, 2008). We adopted methods developed elsewhere for Tpi and Ldh (Dopman et al., 2005), whereas initial Per sequence for the ECB was obtained using degenerate PCR.

DNA from mapping family individuals was extracted from adult legs following using a DNeasy Blood and Tissue kit (QIAGEN, Valencia, CA, USA). Genotyping assays for offspring were developed from segregating polymorphisms and consisted of diagnostic PCR products that amplified polymorphic indels and/or restriction enzyme cut sites (EcoR1, Alu1 and Bsr 1 for Tpi, Per and Ldh, respectively; Supplementary Table S1). Only female offsprings were genotyped and, because female Lepidoptera are the heterogametic sex and possess only one $\mathrm{Z}$ chromosome, one allele per locus was detected. Genotyping results were confirmed by Sanger sequencing $(n \sim 100$ sequenced alleles per marker). One $\mathrm{Z} \times \mathrm{E}$ cross had already been genotyped for $T p i$ and $L d h$ in a prior study (Dopman et al., 2005).

\section{Colocalization of reproductive isolation}

As prior results (Figure 1) and preliminary data suggested evidence for reduced recombination near Tpi in F1 hybrids, we tested for colocalization of traits involved in reproductive isolation. A subset of $\mathrm{Z} \times \mathrm{E}$ backcross offspring was mapped for a major sex-linked factor (named $P d d$ ) controlling diapause emergence time (Glover et al., 1992; Dopman et al., 2005), defined as the time to pupation for over-wintering caterpillars under environmental conditions conducive to breaking diapause. Differences at $P d d$ contribute to temporal isolation between $\mathrm{Z}$ and $\mathrm{E}$ strains in North America by conferring an 30-day shift in emergence and thus adult-mating flights (Wadsworth et al., 2013). Shifts in adult flights between univoltine $\mathrm{Z}$ and bivoltine $\mathrm{E}$ populations eliminate as much as $85 \%$ gene flow in nature (Dopman et al., 2010).

We measured diapause timing variation by inducing and then breaking diapause in recombinant ECB caterpillars. Following earlier studies (Glover et al., 1992; Dopman et al., 2005), diapause was induced by a 12:12 light:dark photoperiod and then broken 35 days later with a 16:8 light:dark cycle. Diapause emergence time was noted every 2 days. As females are hemizygous and $P d d$ is inherited as a Mendelian locus, backcross females show patterns of emergence consistent with either their grandfather (here, E-strain) or grandmother (here, Z-strain; Glover et al., 1992; Dopman et al., 2005). Hence, recombinant backcross females show E-like earlier emergence times and pupate in less than 22 days (range $=8-22$ days, mean $=15.5$ days, s.e. \pm 0.55 ), or they show Z-like later emergence times and pupate in more than 18 days (range = $18-80$, mean $=43.94$ days, s.e. \pm 1.64 ; Glover et al., 1992). 


\section{Higher introgression in collinear regions}

We evaluated patterns of genetic variation among field-caught female ECB: 18 insects were E-strain, 18 were Z-strain and at least one insect was from an outgroup species, the Asian corn borer (ACB, O. furnacalis). ECB caterpillars, pupae or adults were collected over a large geographic range, and thus genetic similarities should be viewed as indicating a combination of ongoing or recent gene exchange. Samples included insects from USA $(n=7$ E-strain and $n=5$ Z-strain from New York; $n=7$ E-strain from North Carolina and $n=4 \mathrm{Z}$-strain from North Carolina; $n=4$ Z-strain from Iowa), Italy ( $n=4$ E-strain and $n=3$ $Z$-strain) and Hungary ( $n=2 \mathrm{Z}$-strain). Moths were classified into strain by diagnostic gas chromatographic profiles of female sex-pheromone blend (that is, 99:1 versus 3:97 ratio of E/Z11-14:OAc; for example, Dopman et al., 2004) and/or by genotyping the $p g F A R$ locus.

Molecular markers were developed from genes that were evenly distributed across the B. mori sex chromosome. Using ECB transcripts that were developed from a separate study, reciprocal BLASTs were performed to obtain ECB- $B$. mori gene pairs with predicted locations on the ECB sex chromosome (B. mori nucleotide CDS, BLASTn, $e$-value $<1 \mathrm{e}-40)$. ECB transcripts were then searched with tBLASTx against the matching $B$. mori genomic sequence to identify predicted introns. Primer pairs for 23 loci (Supplementary Table S2) that had interlocus intervals ranging from 0.1 to $2.5 \mathrm{Mb}$ (mean $=0.96 \mathrm{Mb}$, s.e. \pm 0.16$)$ were designed to amplify ECB introns using PrimerBlast (NCBI). Sequences from field-caught insects were aligned and edited by eye using Genious Pro 5.5 (Biomatters Inc., San Francisco, CA, USA). Markers were named based on Bombyx gene identifiers.

Measures of genetic variation $\left(\pi, \theta_{w}\right)$, genetic differentiation $\left(d_{\mathrm{a}}, d_{\mathrm{xy}}, F_{\mathrm{ST}}\right.$ (Hudson et al., 1992), $S_{\mathrm{nn}}$ (Hudson, 2000)) and the allele-frequency spectrum (Tajima's $D$ (Tajima, 1989b), Fay and Wu's $H$ (Fay and Wu, 2000)) were calculated using DnaSP (Rozas et al., 2003). The genealogical sorting index ( $g s i$; Cummings et al., 2008) was calculated to quantify the degree of exclusive ancestry of Z- and E-strain moths. gsi ranges from 0 (no exclusivity) to 1 (monophyletic), and was calculated in R (Team RDC, 2013) on the Tufts highperformance computing research cluster using the genealogicalSorting library (www.genealogicalsorting.org). Genealogies were constructed using neighborjoining with a Tamura-Nei 93 model of evolution to calculate distances. Statistical significance of $g s i$ was assessed through permutation tests $(n=10000)$ on each of 1000 bootstrap replicates for a total of 10000000 replicated genealogies. Indel polymorphisms were considered a fifth base when calculating genetic differentiation and genetic exclusivity. All figures were constructed using the ggplot2 library (Wickham, 2009) in R.

\section{RESULTS}

\section{Recombination suppression}

We used three-point crosses within- and between-strain to test for evidence of suppressed recombination associated with a highfrequency chromosomal rearrangement between $\mathrm{Z}$ and $\mathrm{E}$ strains of ECB. No significant differences in recombination frequency were observed among different within-strain backcross families $(Z \times Z$, $n=2$ families) or among between-strain backcross families $(Z \times E$, $n=7$ families) and therefore families were combined for each backcross type. Within strain, we observed six recombinants between the Tpi and Per pair (7\%, $n=86$ offsprings), six between the Per and $L d h$ pair (7\%, $n=86$ offsprings) and twelve between Tpi and $L d h$ (14\%, $n=86$ offsprings; Figure 2a). Hence, in agreement with that found in B. mori, the predicted gene order within the Z-strain of ECB is TpiPer-Ldh (Figure 2b).

Between-strain crosses revealed heterogeneity in recombination frequency compared with that seen within-strain (Figure 2a). ZxE crosses yielded slightly elevated recombination frequencies for the Per$L d h$ gene pair (seven recombinants, 9.1\%, $n=77$ offsprings; Fisher's exact test, $P$-value $=0.7739$ ) and slightly reduced recombination between $T p i$ and $L d h$ (seven recombinants, 9.1\%, $n=77$ offsprings; Fisher's exact test, $P$-value $=0.4643$ ). However, a statistically significant suppression in recombination was observed between Tpi and Per.
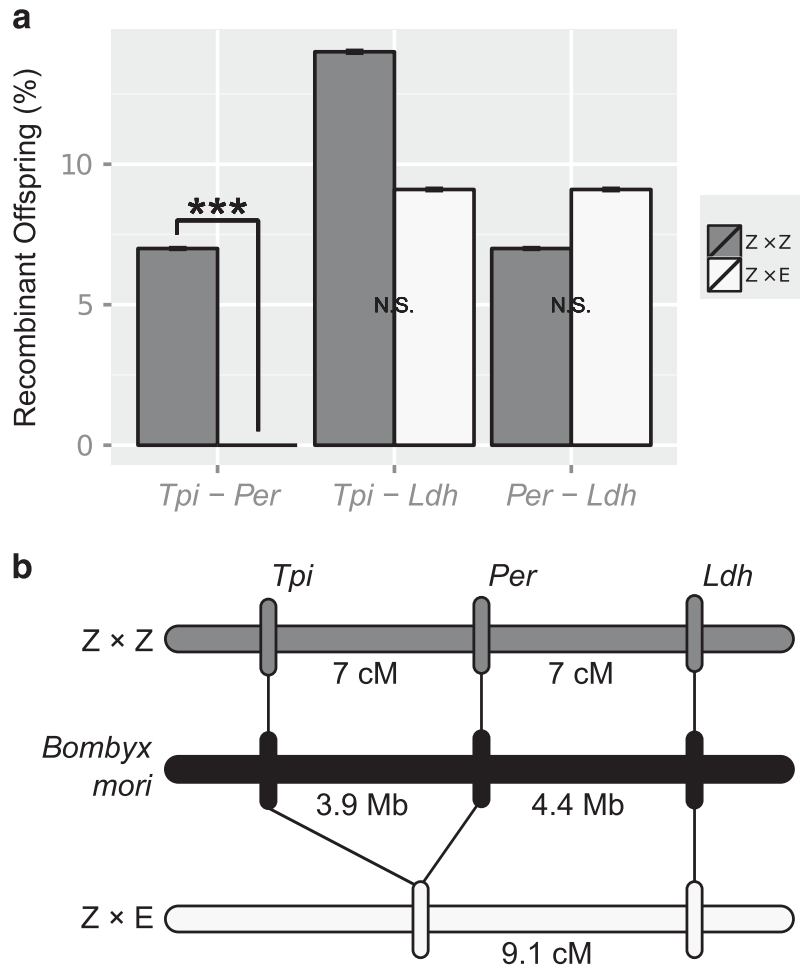

Figure 2 Suppressed recombination on the sex chromosome in ECB hybrids. (a) Percent recombinant offspring between Tpi, Per and $L d h$ in within-strain $(Z \times Z)$ and between-strain $(Z \times E)$ crosses. (b) Inferred linear order of markers in Bombyx mori and the two ECB crosses. ${ }^{* * *} P$-value $<0.001$.

Zero recombinants were found in over 600 offsprings $(n=666$ offsprings; Fisher's exact test, $P$-value $=1.91 \mathrm{e}-06$ ).

\section{Colocalization of reproductive isolation}

We phenotyped a subset of backcross offspring to determine the linkage relationship between the nonrecombining Tpi-Per gene pair and $P d d$. Diapause emergence timing of 333 hemizygous females from backcross families ranged from 2 to 100 days; yet, a clear bimodal distribution emerged (Figure 3). Eight offspring (2.4\%) had emergence times that fell within the period of overlap of parental $\mathrm{Z}$ and $\mathrm{E}$ strains (day 18-22) and their Pdd genotype was considered indeterminate. The remaining 325 offspring had emergence times consistent with pure-strain insects. Of these, one female with E-strain markers at the Tpi-Per gene pair displayed Z-strain like diapause and emerged later, and one female with Z-strain markers displayed E-strain like diapause and emerged earlier. Thus, two females $(0.62 \%)$ were apparent recombinants between the Tpi-Per gene pair and the Pdd locus. In the other 323 offspring (99.38\%), Pdd was perfectly associated with their genotype at the Tpi-Per gene pair.

\section{Higher introgression in collinear regions}

We characterized genetic variation for 23 loci to test for differences in patterns of genetic variation between loci with predicted locations inside (referred to as 'rearranged') and outside (referred to as 'collinear') of the nonrecombining Tpi-Per chromosomal region. A total of 779 sequences were obtained, resulting in $\sim 9 \mathrm{~kb}$ of aligned data and 224 segregating sites. Seven loci fell within the Tpi-Per interval and 16 were outside ( 8 upstream and 8 downstream).

We tested for variation in patterns of genetic differentiation using four different indices. Rearranged loci exhibited statistically 
significantly elevated evolutionary divergence for both $F_{\mathrm{ST}}$ (median rearranged $=0.14990$, median collinear $=0.06418, W=88, P$-value $=$ $0.01764)$ and $S_{\text {nn }}$ (median rearranged $=0.6569$, median collinear $=$ $0.5742, W=90, P$-value $=0.01258$; Figure 4$)$. Similarly, the number of net substitutions per site between strains $\left(d_{\mathrm{a}}\right.$, median rearranged $=$ $0.09 \%$, median collinear $=0.029 \%, W=74, P$-value $=0.121)$ and the average number of substitutions per site between strains $\left(d_{\mathrm{xy}}\right.$, median rearranged $=0.76 \%$, median collinear $=0.66 \%, W=54, P$-value $=$ 0.5647 ) were both higher for rearranged loci (Figure 4), although these differences were not statistically significant.

We also used gsi to explore gene tree topology measures of genetic differentiation (Table 1). Loci with significant values of gsi ranged from 0.01 to 0.68 with an average of 0.22 . E-strain ECB had seven loci with significant genealogical exclusivity, whereas the Z-strain had 15. There were no significant differences in the magnitude of gsi for rearranged and collinear loci $(t=-1.3504$, degrees of freedom $=$ 19.019, $P$-value $=0.1927$ ); however, all rearranged loci possessed significant genealogical exclusivity in either one or both strains, in contrast to $\sim 50 \%$ of collinear loci (Fisher's exact test, $P$ value $=0.0574)$.

To evaluate patterns of nucleotide polymorphism, we compared $\pi$ and $\theta_{w}$ between groups. Average genetic variation for rearranged loci was approximately one-half of that for collinear loci (Table 2), although only statistically significant (or trending) for E-strain moths (mean rearranged $\pi=0.386 \%$, mean collinear $\pi=0.812 \%$, $t=-1.7158$, degrees of freedom $=16.839, P$-value $=0.05227$; mean $\theta_{w}=0.347 \%$, mean collinear $\theta_{w}=0.916 \%, t=-2.3966$, degrees of freedom $=15.098, P$-value $=0.01496)$. For both strains Tajima's $D$ showed a slight skew toward an excess of intermediate-frequency alleles compared with low-frequency alleles in rearranged loci (that is, more positive values; Table 2), whereas Fay and Wu's $H$ revealed a relative excess of high-frequency derived alleles compared with intermediate-frequency alleles (that is, more negative values; Table 2). However, neither estimate was statistically significant.

\section{DISCUSSION}

Although our post-genomic age has produced billions of base pairs of DNA sequence data for many organisms across the speciation continuum, we often lack adequate biological explanations for the evolution of fundamental genomic features such as genetic divergence. Inversions are thought to have far-reaching impacts on evolutionary divergence during speciation, especially when divergence occurs in the face of gene flow. However, demonstrating the importance of inversions to the speciation process requires evidence for (i) recombination suppression in hybrid offspring, (ii) localization of barrier or adaptation loci in rearranged regions and (iii) enhanced sequence divergence (Faria and Navarro, 2010). In this study, we combined genetic crosses (Figure 2), phenotyping of traits underlying reproductive isolation (Figure 3 ) and observational studies of DNA polymorphism in wild populations (Figure 4, Table 1) to show that $Z$ and E strains of ECB meet all three criteria. Although we cannot physically confirm the presence of an inversion yet through cytology, these results are consistent with the presence of an inversion that has contributed to accumulation of ecologically adaptive alleles and genetic

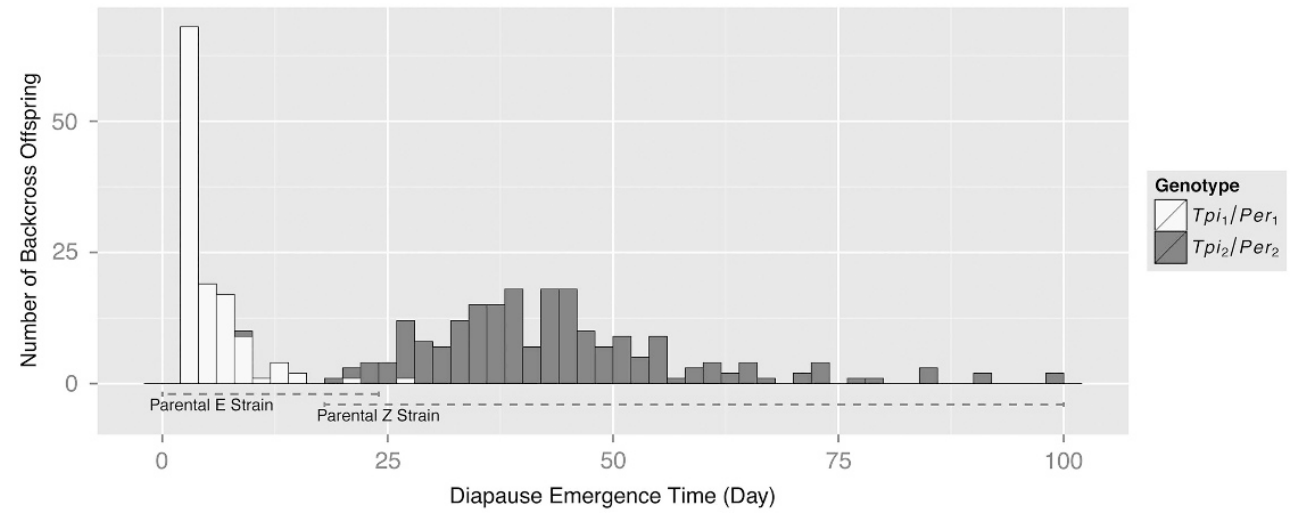

Figure 3 Diapause emergence time maps to a putative sex-linked inversion. Bimodal distribution of diapause emergence time for female backcross offspring

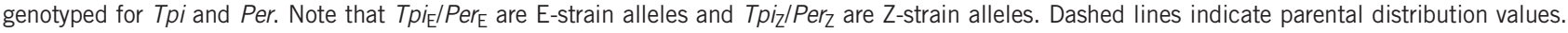
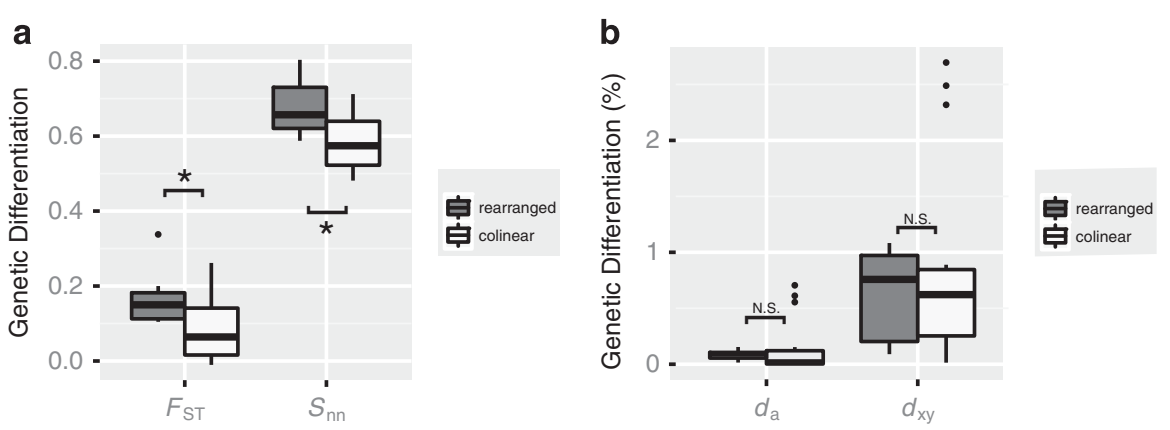

Figure 4 Elevated evolutionary divergence between ECB strains. (a) $F_{\mathrm{ST}}$ and $S_{\mathrm{nn}}$. (b) $d_{\mathrm{a}}$ and $d_{\mathrm{xy}}$. ${ }^{*} P$-value $<0.05$. 
Table 1 Genetic exclusivity between ECB strains for colinear and rearranged loci

\begin{tabular}{|c|c|c|c|c|c|}
\hline Location & Locus & $g s i_{E}$ & P-value & $g s_{i Z}$ & P-value \\
\hline Rearranged & Tpi & 0.14 & N.S. & 0.26 & $* * *$ \\
\hline Rearranged & Per & 0.11 & N.S. & 0.21 & * \\
\hline Rearranged & 680 & 0.28 & $* *$ & 0.35 & $* * *$ \\
\hline Rearranged & 524 & 0.18 & N.S. & 0.35 & $* * *$ \\
\hline Rearranged & 710 & 0.17 & N.S. & 0.26 & $* *$ \\
\hline Rearranged & 1368 & 0.19 & N.S. & 0.29 & ** \\
\hline Rearranged & 683 & 0.18 & * & 0.12 & N.S \\
\hline Colinear & 2071 & 0.23 & N.S. & 0.23 & N.S \\
\hline Colinear & 2076 & 0.13 & N.S. & 0.17 & N.S \\
\hline Colinear & 2104 & 0.1 & N.S. & 0.12 & N.S \\
\hline Colinear & 2005 & 0.17 & N.S. & 0.28 & * \\
\hline Colinear & 622 & 0.14 & N.S. & 0.2 & N.S \\
\hline Colinear & 643 & 0.26 & $* * *$ & 0.23 & $* * *$ \\
\hline Colinear & 647 & 0.33 & $* *$ & 0.32 & $* *$ \\
\hline Colinear & 563 & 0.02 & N.S. & 0.5 & $* * *$ \\
\hline Colinear & 9691 & 0.68 & $* * *$ & 0.52 & $* * *$ \\
\hline Colinear & 13318 & 0.01 & N.S. & 0.23 & $* *$ \\
\hline Colinear & 13333 & 0.2 & N.S. & 0.22 & N.S \\
\hline Colinear & 8010 & 0.28 & $* * *$ & 0.33 & $* * *$ \\
\hline Colinear & 12292 & 0.08 & N.S. & 0.04 & N.S \\
\hline Colinear & 13832 & 0.19 & $* *$ & 0.28 & $* * *$ \\
\hline Colinear & 587 & 0.12 & N.S. & 0.29 & $* *$ \\
\hline Colinear & 2781 & 0.07 & N.S & 0.12 & N.S \\
\hline
\end{tabular}

${ }^{* * *} P$-value $<0.001 ; * * P$-value $<0.01 ;{ }^{*} P$-value $<0.05$. N.S. not significant.

asignificance determined by permutation $(n=10000)$ of bootstrap replicated $(n=1000)$.

Table 2 Patterns of genetic variation among rearranged and colinear loci

\begin{tabular}{lccrrrrr}
\hline & Loci & Length $^{\mathrm{a}}$ & $S^{\mathrm{b}}$ & $\pi^{\mathrm{c}}(\%)$ & $\theta^{\mathrm{d}}(\%)$ & \multicolumn{1}{c}{$D^{\mathrm{e}}$} & \multicolumn{1}{c}{$H^{\mathrm{f}}$} \\
\hline E Strain & & & & & & & \\
$\quad$ Rearranged & 7 & 3541 & 47 & 0.386 & 0.347 & 0.214 & -0.229 \\
$\quad$ Colinear & 16 & 5172 & 107 & 0.812 & 0.916 & -0.492 & -0.178 \\
$\quad P$-value & & & & 0.052 & 0.015 & 0.888 & 0.473 \\
& & & & & & & \\
$Z$ Strain & & & & & & & \\
$\quad$ Rearranged & 7 & 3549 & 60 & 0.486 & 0.514 & -0.165 & -3.266 \\
$\quad$ Colinear & 16 & 5172 & 106 & 0.812 & 0.849 & -0.379 & -0.883 \\
$P$-value & & & & 0.127 & 0.102 & 0.646 & 0.131 \\
\hline
\end{tabular}

aBase pairs. ${ }^{b}$ Segregating sites. ${ }^{c}$ Heterzygosity from average number of differences per site.

dHeterzygosity from number of polymorphic sites. ${ }^{\mathrm{e} T a j i m a, 1989 a, b .}{ }^{\mathrm{F} F a y}$ and $\mathrm{Wu}, 2000$.

differentiation across roughly $20 \%$ of the ECB sex chromosome $(\sim 4 \mathrm{Mb})$.

\section{Inversions and reproductive isolation}

A major appeal of chromosomal rearrangement models of speciation is their ability to help explain apparent examples of speciation with gene flow (Pinho and Hey, 2010). Indeed, a serious theoretical weakness of nonallopatric speciation is that recombination randomizes associations between genes conferring local adaptation (hybrid unfitness) and assortative mating (Felsenstein, 1981; Gavrilets, 2003; Butlin, 2005). Although the specifics of chromosomal rearrangement models of speciation differ (Noor et al., 2001; Rieseberg, 2001; Navarro and Barton, 2003; Kirkpatrick and Barton, 2006; Faria and Navarro, 2010), a common feature is the removal of this antagonism and a buildup of adaptation and barrier loci within rearrangements during a nonallopatric phase(s) that then allows for reinforcement and the completion of speciation. Thus, a critical prediction for speciation with gene flow is that rearrangements such as inversions should be present and contain multiple prezygotic and/or postzygotic barrier loci with major effects on reproductive isolation. Determining the degree to which these expectations are borne out in nature is challenging. First, we have limited data on quantitative measures of reproductive isolation, defined as $R I=1$ - (between species fitness) $\div$ (within species fitness) and ranging from 0 (no effect) to 1 (complete RI; Ramsey et al., 2003; Coyne and Orr, 2004). Second, the genomic positions of the underlying barrier loci is often unknown. Monkeyflowers are a rare exception, in which loci of large reproductive isolation effect that contribute to floral traits, flowering time and hybrid male sterility map to chromosomal rearrangements in the Mimulus genome (Ramsey et al., 2003; Lowry and Willis, 2010; Fishman et al., 2013).

The putative sex-linked inversion we document in Ostrinia appears consistent with the large-effect, multicomponent prediction of chromosomal rearrangement models of speciation. First, multiple 'speciation phenotypes' (Shaw and Mullen, 2011) in ECB have known sex-linked factors, including male pheromone response and diapause timing (McLeod, 1978; Dopman et al., 2004; 2005; Wanner et al., 2010; Ikten et al., 2011). Second, a recent study indicated that large isolation effect sizes are common between ECB strains $(R I \geqslant 0.5$ for 4 of 7 forms of isolation; Dopman et al., 2010). Given this prior work and the large size of the putative inversion $(7 \mathrm{cM}$ or $\sim 4 \mathrm{Mb}$; Figure $2 \mathrm{~b}$ ), there would appear to be good reason to expect diverse and potent barrier loci in an inversion on the ECB sex chromosome. Indeed, we found that only two of 325 offspring were recombinants $(0.62 \%)$ between the nonrecombining region (Tpi-Per loci, Figure $2 \mathrm{~b}$ ) and the factor(s) determining diapause emergence time (Pdd; Figure 3). Thus, a major cause of temporal isolation (mean $R I=0.65$ ) is likely inside or near the chromosomal rearrangement. Similarly, the sex-linked ORs are known to underlie strain-specific male antennal response to $\mathrm{Z}$ versus $\mathrm{E}$-strain female pheromones (that is, 3:97 versus 99:1 ratio of E/Z11-14:OAc) and they are tightly linked to Tpi and thus the sex-linked rearrangement (Wanner et al., 2010; Lassance et al., 2011). Hence, a rearrangement may have promoted coupling between multiple genes conferring ecological $(P d d)$ and behavioral (ORs) divergence, thereby facilitating speciation. Clearly, there is a need for formal experimentation to determine whether chromosomal rearrangements in Ostrinia and in other systems harbor multiple loci of large isolation effect that contribute to varied components of reproductive isolation.

\section{Evolution of genetic divergence}

Results from several systems including Ostrinia and Drosophila (McGaugh and Noor, 2012) imply high genetic divergence within inversions and minimal divergence outside of them, and a reciprocal relationship for polymorphism. Such patterns may be common in the early stages of speciation because of the coupling of selection and recombination. Globally beneficial mutations and adaptive barrier alleles that arise within fixed inversions are expected to get trapped and cannot easily migrate between lineages (Navarro and Barton, 2003). Further, according to the Kirkpatrick-Barton model (Kirkpatrick and Barton, 2006), selective establishment and fixation of inversions occurs because locally adaptive alleles at loci within inversions (for example, underlying ecological and behavioral divergence) cannot recombine with chromosomes from other populations and thus they are favored because they do not suffer the 'Achilles' heel' of being located on the same chromosome as immigrant disadvantageous allele(s). Hence, genetic hitch-hiking associated with either 
selective fixation of inversions or their internal mutations might lead to inversion-specific reductions in polymorphism within one or both lineages, while also creating enhanced differentiation for relative divergence measures that are sensitive to within lineage variation (for example, $F_{\mathrm{ST}}$ Charlesworth, 1998).

An additional role of suppressed recombination for evolutionary divergence involves repeated bouts of gene exchange and disruptive selection. Specifically, barrier loci within inversions under the Navarro-Barton model (Navarro and Barton, 2003) might produce a molecular signal of differentiation that is spread across the inversion or at least near its breakpoints (Rieseberg, 2001; Noor et al., 2001; Strasburg et al., 2009; McGaugh and Noor, 2012) because of selective purging of maladapted (and nonrecombining) regions encompassed by inversions upon their introduction into sister taxa. Such a process might be analogous to 'differential introgression,' in which gene flow is restricted at individual barrier loci but is high elsewhere (Barton and Bengtsson, 1986; Wu, 2001; West and Via, 2008; Harrison, 2012). Over the long term, differential introgression is expected to produce specific signatures. For example, increases in absolute genetic divergence (for example, $d_{\mathrm{xy}}$ ) as new mutations arise and fix independently in daughter taxa (Charlesworth et al., 1997; Charlesworth, 1998; Nachman and Payseur, 2012; Cruickshank and Hahn, 2014).

Despite limitations of our current sampling scheme (18 samples per strain from both allopatric and sympatric localities), results from Ostrinia suggest that selection and suppressed recombination were important for evolutionary divergence. Compared with 'collinear' loci, patterns of genetic variation at 'rearranged' loci are consistent with a selective sweep(s), including 50\% lower genetic variation (primarily in E-strain moths; Table 2) and 2-3× greater differentiation for measures that are sensitive to selection at linked sites $\left(F_{\mathrm{ST}}\right.$ and $d_{\mathrm{a}}$; Figure 4). Support for differential introgression is less obvious. The putative inversion shows modest increases in absolute divergence $\left(1.15 \times\right.$ greater values of $\left.d_{\mathrm{xy}}\right)$ and also genealogical exclusivity $(1.14 \times$ greater values of $\left.S_{\mathrm{nn}}\right)$. Moreover, although patterns in the allelefrequency spectrum $(D$ and $H)$ are consistent with rare migration within the inversion and higher migration elsewhere (Table 2; Tajima, 1989a; Simonsen et al., 1995; Przeworski, 2002; Fay and Wu, 2005), these changes are relatively minor. Lack of genetic evidence for differential introgression runs counter to observations in nature. An appreciable number of hybrid offsprings occur at sampled sympatric localities in New York (5-15\%; Dopman et al., 2010; Coates et al., 2013) and yet differentiation between $Z$ and $E$ strains at Tpi has been maintained at these sites for more than 40 generations (Glover et al., 1991; Dopman et al., 2005; Dopman, 2011). Disruptive selection following introgression of the nonrecombining region that includes Tpi might be important in maintaining divergence at New York localities, where sweeps and incomplete lineage sorting alone cannot easily account for patterns of nucleotide variation across loci. Equivocal genetic support for differential introgression should not be surprising because statistical power is limited during early stages of speciation (for example, for $d_{\mathrm{xy}}$; Cruickshank and Hahn, 2014). Hence, lineages that are in the early stages of divergence like ECB strains may be too closely related to show strong statistical signs of differential gene flow even at genome regions where it occurs.

More extensive investigation of Ostrinia moths and other systems might validate the scenario we propose in which evolutionary divergence during speciation occurs by two complementary but distinct mechanisms - one driving the transient origin of differentiation through local adaptation and selection on linked sites, and one determining its stable persistence through reproductive isolation and/ or differential introgression (see also Dopman, 2011; Nachman and
Payseur, 2012; Cruickshank and Hahn, 2014). If an inversion is present as a low-frequency polymorphism in allopatry and greatly differentiates lineages only in geographic regions where they co-occur (for example, as in the 'mixed-geographic model' (Feder et al., 2011)), this might suggest a direct role of recombination suppression in promoting evolutionary divergence by local adaptation and selective sweeps (for example, to eliminate the creation of less fit hybrids or to keep high fitness loci together). In contrast, the presence of an older, high-frequency inversion that differentiates lineages regardless of geography could indicate an indirect role of recombination suppression in facilitating accumulation of additional genetic changes once inversions establish or fix. That is, the rearrangement could protect chromosomal regions from gene flow and allow for additional genetic changes to accrue (for example, through local sweeps of internal beneficial mutations or differential introgression of the region encompassed by the rearrangement). Broader comparisons between recently sympatric Z and E strains of Ostrinia living in North America and their allopatric, European ancestors may help distinguish between direct and indirect roles of recombination suppression during divergence.

\section{CONCLUSIONS}

Our studies of recombination, reproductive isolation and genetic variation in the wild imply that structural mutations have facilitated speciation with gene flow between $\mathrm{Z}$ and $\mathrm{E}$ strains of ECB. Our results add to the limited number of studies that provide empirical support for this notion, including apple maggot flies (Feder et al., 2003; 2005; Michel et al., 2010; Powell et al., 2013), fruit flies (Noor et al., 2001; Kulathinal et al., 2009), stickleback fishes (Kitano et al., 2009), sunflowers (Rieseberg, 2001; Strasburg et al., 2009) and monkeyflowers (Lowry and Willis, 2010; Fishman et al., 2013). Our finding that structural mutations contribute to speciation may be the first of many for Lepidoptera. The rate of rearrangement in lepidopteran genomes appears to be one of the fastest among eukaryotes at $\sim 2$ breakages/Mb/My, or $\sim 3 \times$ faster than nematodes and more than an order of magnitude faster than flies, mammals and plants (Ranz et al., 2001; Coghlan and Wolfe, 2002; d'Alencon et al., 2010). As lepidopterans (and nematodes) have diffusely organized centromeres (holocentric), these high evolutionary rates may stem from an increased likelihood of reintegration of double-strand break fragments (d'Alencon et al., 2010). Hence, tens or even hundreds of fixed rearrangements could characterize moth or butterfly species pairs that diverged just several hundred thousand years ago, implying that structural mutations may have had a disproportionate role over the evolutionary history of the second largest order of insects, and may commonly promote adaptive divergence and speciation in moths and butterflies.

\section{DATA ARCHIVING}

Data available from the Dryad Digital Repository: http://doi.org/ $10.5061 /$ dryad.2vg05

\section{CONFLICT OF INTEREST}

The authors declare no conflict of interest.

\section{ACKNOWLEDGEMENTS}

We thank Charles Linn for insect stocks, Jean-Marc Lassance for initial Per primers and Genevieve Kozak for comments on an early version of the manuscript. The study was supported by the USDA (EBD: 2010-65106-20610), NSF (EBD: DEB-1257251; CBW: 2011116050) and Tufts University (XL: 
Summer Scholar Program). We thank Jeff Feder, Aurora Ruiz-Herrera and three anonymous referees for suggestions on this final version.

Barton N, Bengtsson BO (1986). The barrier to genetic exchange between hybridising populations. Heredity 57: 357-376.

Butlin RK (2005). Recombination and speciation. Mol Ecol 14: 2621-2635.

Caffrey D (1927). A Progress Report on the Investigations of the European Corn Borer. Governmental Printing Office: Washington, DC.

Charlesworth B (1998). Measures of divergence between populations and the effect of forces that reduce variability. Mol Biol Evol 15: 538-543.

Charlesworth B, Nordborg M, Charlesworth D (1997). The effects of local selection, balanced polymorphism and background selection on equilibrium patterns of genetic diversity in subdivided populations. Genet Res 70: 155-174.

Coates BS, Johnson H, Kim K-S, Hellmich RL, Abel CA, Mason C et al. (2013). Frequency of hybridization between Ostrinia nubilalis E-and Z-pheromone races in regions of sympatry within the United States. Ecol Evol 3: 2459-2470.

Coghlan A, Wolfe KH (2002). Fourfold faster rate of genome rearrangement in nematodes than in Drosophila. Genome Res 12: 857-867.

Coyne JA, Orr HA (2004). Speciation1st edn. Sinauer Associates Inc: Sunderland, MA

Cruickshank TE, Hahn MW (2014). Reanalysis suggests that genomic islands of speciation are due to reduced diversity, not reduced gene flow. $\mathrm{Mol} E \mathrm{Col} 23$ : 3133-3175.

Cummings MP, Neel MC, Shaw KL (2008). A genealogical approach to quantifying lineage divergence. Evolution 62: 2411-2422.

d'Alencon E, Sezutsu H, Legeai F, Permal E, Bernard-Samain S, Gimenez S et al. (2010). Extensive synteny conservation of holocentric chromosomes in Lepidoptera despite high rates of local genome rearrangements. Proc Natl Acad Sci USA 107: 7680-7685.

Dopman EB (2011). Genetic hitchhiking associated with life history divergence and colonization of North America in the European corn borer moth. Genetica 139: 565-573.

Dopman EB, Bogdanowicz SM, Harrison RG (2004). Genetic mapping of sexual isolation between $\mathrm{E}$ and $\mathrm{Z}$ pheromone strains of the european corn Borer (Ostrinia nubilalis). Genetics 167: 301-309.

Dopman EB, Pérez L, Bogdanowicz SM, Harrison RG (2005). Consequences of reproductive barriers for genealogical discordance in the European corn borer. Proc Natl Acad SCi USA 102: 14706-14711.

Dopman EB, Robbins PS, Seaman A (2010). Components of reproductive isolation between North American pheromone strains of the European Corn Borer. Evolution 64: 881-902.

Faria R, Navarro A (2010). Chromosomal speciation revisited: rearranging theory with pieces of evidence. Trends Ecol Evol 25: 660-669.

Fay J, Wu C-I (2000). Hitchhiking under positive Darwinian selection. Genetics 155: 1405-1413

Fay JC, Wu C-I (2005). Detecting hitchhiking from patterns of DNA polymorphism. In Nurminsky D (ed) Selective Sweep. Kluwer Academic/Plenum Publishers: New York, NY.

Feder J, Roethele J, Filchak K, Niedbalski J (2003). Evidence for inversion polymorphism related to sympatric host race formation in the apple maggot fly, Rhagoletis pomonella. Genetics 163: 939-953.

Feder JL, Egan SP, Nosil P (2012). The genomics of speciation-with-gene-flow. Trends Genet 28: 342-350.

Feder JL, Gejji R, Powell THQ, Nosil P (2011). Adaptive chromosomal divergence driven by mixed geographic mode of evolution. Evolution 65: 2157-2170.

Feder JL, Gejji R, Yeaman S, Nosil P (2012). Establishment of new mutations under divergence and genome hitchhiking. Philos TR Soc B 367: 461-474.

Feder JL, Xie X, Rull J, Velez S, Forbes A, Leung B et al. (2005). Mayr, Dobzhansky, and Bush and the complexities of sympatric speciation in Rhagoletis. P Natl Acad Sci USA 102 (Suppl 1): 6573-6580.

Felsenstein J (1981). Skepticism towards Santa Rosalia, or why are there so few kinds of animals? Evolution 35: 124-138.

Fishman L, Stathos A, Beardsley PM, Williams CF, Hill JP (2013). Chromosomal rearrangements and the genetics of reproductive barriers in Mimulus (monkey flowers). Evolution 67: 2547-2560.

Gavrilets S (2003). Perspective: models of speciation: what have we learned in 40 years? Evolution 57: 2197-2215.

Glover TJ, Knodel JJ, Robbins PS, Eckenrode CJ, Roelofs WL (1991). Gene flow among three races of European corn borers (Lepidoptera: Pyralidae) in New York State. Environ Entomol 20: 1356-1362.

Glover TJ, Robbins PS, Eckenrode CJ, Roelofs WL (1992). Genetic control of voltinism characteristics in European corn borer races assessed with a marker gene. Arch Insect Biochem 20: 107-117.

Harrison RG (2012). The language of speciation. Evolution 66: 3643-3657.

Hudson RR (2000). A new statistic for detecting genetic differentiation. Genetics 155: 2011-2014.

Hudson RR, Slatkin M, Maddison WP (1992). Estimation of levels of gene flow from DNA sequence data. Genetics 132: 583-589.
Ikten C, Skoda SR, Hunt TE, Molina-Ochoa J, Foster JE (2011). Genetic variation and inheritance of diapause induction in two distinct voltine ecotypes of Ostrinia nubilalis (Lepidoptera: Crambidae). Ann Entomol Soc Am 104: 567-575.

International Silkworm Genome Consortium (2008). The genome of a lepidopteran model insect, the silkworm Bombyx mori. Insect Biochem Molec 38: 1036-1045.

Kirkpatrick M (2010). How and why chromosome inversions evolve. PLoS Biol 8: e1000501.

Kirkpatrick M, Barton N (2006). Chromosome inversions, local adaptation and speciation. Genetics 173: 419-434

Kitano J, Ross JA, Mori S, Kume M, Jones FC, Chan YF et al. (2009). A role for a neo-sex chromosome in stickleback speciation. Nature 461: 1079-1083.

Klun JA, Cooperators (1975). Insect sex pheromones: Intraspecific pheromonal variability of Ostrinia nubilalis in North America and Europe. Environ Entomol 4: 891-894.

Kroemer JA, Coates BS, Nusawardani T, Rider SD, Fraser LM, Hellmich RL (2011). A rearrangement of the $Z$ chromosome topology influences the sex-linked gene display in the European corn borer. Ostrinia nubilalis. Mol Genet Genomics 286: 37-56.

Kulathinal RJ, Stevison LS, Noor MAF (2009). The genomics of speciation in Drosophila: diversity, divergence, and introgression estimated using low-coverage genome sequencing. PLoS Genet 5: e1000550.

Lassance J-M, Bogdanowicz SM, Wanner KW, Löfstedt C, Harrison RG (2011). Gene genealogies reveal differentiation at sex pheromone olfactory receptor loci in pheromone strains of the European Corn Borer, Ostrinia nubilalis. Evolution 65: 1583-1593.

Lassance J-M, Groot AT, Liénard MA, Antony B, Borgwardt C, Andersson F et al. (2010). Allelic variation in a fatty-acyl reductase gene causes divergence in moth sex pheromones. Nature 466: 486-489.

Lowry DB, Willis JH (2010). A widespread chromosomal inversion polymorphism contributes to a major life-history transition, local adaptation, and reproductive isolation. PLOS Biol 8: e1000500.

Malausa T, Leniaud L, Martin J-F, Audiot P, Bourguet D, Ponsard S et al. (2007). Molecular differentiation at nuclear loci in French host races of the European corn borer (Ostrinia nubilalis). Genetics 176: 2343-2355.

McGaugh SE, Noor MAF (2012). Genomic impacts of chromosomal inversions in parapatric Drosophila species. Philos T R Soc B 367: 422-429.

McLeod D (1978). Genetics of diapause induction and termination in the European corn borer, Ostrinia nubilalis (Lepidoptera: Pyralidae), in southwestern Ontario. Can Entomol 110: 1351-1353.

Michel AP, Sim S, Powell THQ, Taylor MS, Nosil P, Feder JL (2010). Widespread genomic divergence during sympatric speciation. Proc Natl Acad Sci USA 107: 9724-9729.

Nachman MW, Payseur BA (2012). Recombination rate variation and speciation: theoretical predictions and empirical results from rabbits and mice. Philos $T R$ Soc $B$ 367: 409-421.

Navarro A, Barton N (2003). Accumulating postzygotic isolation genes in parapatry: a new twist on chromosomal speciation. Evolution 57: 447-459.

Noor MA, Grams KL, Bertucci LA, Reiland J (2001). Chromosomal inversions and the reproductive isolation of species. Proc Natl Acad Sci USA 98: 12084-12088.

Noor MAF, Bennett SM (2009). Islands of speciation or mirages in the desert? Examining the role of restricted recombination in maintaining species. Heredity 103: 439-444.

Pinho C, Hey J (2010). Divergence with gene flow: models and data. Annu Rev Ecol Evol Syst 41: 215-230.

Powell THQ, Hood GR, Murphy MO, Heilveil JS, Berlocher SH, Nosil P et al. (2013). Genetic divergence along the speciation continuum: the transition from host race to species in rhagoletis (Diptera: Tephritidae). Evolution 67: 2561-2576.

Pringle EG, Baxter SW, Webster CL, Papanicolaou A, Lee SF, Jiggins CD (2007). Synteny and chromosome evolution in the lepidoptera: evidence from mapping in Heliconius melpomene. Genetics 177: 417-426.

Przeworski M (2002). The signature of positive selection at randomly chosen loci. Genetics 160: 1179-1189.

Ramsey J, Bradshaw H Jr, Schemske D (2003). Components of reproductive isolation between the monkeyflowers Mimulus lewisii and M. cardinalis (Phrymaceae). Evolution 57: 1520-1534.

Ranz JM, Casals F, Ruiz A (2001). How malleable is the eukaryotic genome? Extreme rate of chromosomal rearrangement in the genus Drosophila. Genome Res 11: 230-239.

Rieseberg LH (2001). Chromosomal rearrangements and speciation. Trends Ecol Evol 16: 351-358.

Roelofs W, Glover T, Tang XH, Sreng I, Robbins P, Eckenrode C et al. (1987). Sex pheromone production and perception in European corn borer moths is determined by both autosomal and sex-linked genes. Proc Natl Acad Sci USA 84: 7585-7589.

Rozas J, Sánchez-DelBarrio JC, Messeguer X, Rozas R (2003). DnaSP, DNA polymorphism analyses by the coalescent and other methods. Bioinformatics 19: 2496-2497.

Shaw KL, Mullen SP (2011). Genes versus phenotypes in the study of speciation. Genetica 139: 649-661.

Simonsen KL, Churchill GA, Aquadro CF (1995). Properties of statistical tests of neutrality for DNA polymorphism data. Genetics 141: 413-429.

Smith HE (1920). Broom Corn, the probable host in which Pyrausta nubilalis, Hubn., reached America. J Econ Entomol 13: 425-430.

Storz JF (2005). Using genome scans of DNA polymorphism to infer adaptive population divergence. $\mathrm{Mol}$ Ecol 14: 671-688.

Strasburg JL, Scotti-Saintagne C, Scotti I, Lai Z, Rieseberg LH (2009). Genomic patterns of adaptive divergence between chromosomally differentiated sunflower species. Mol Biol Evol 26: 1341-1355.

Tajima F (1989a). DNA polymorphism in a subdivided population: the expected number of segregating sites in the two-subpopulation model. Genetics 123: 229-240. 
Tajima F (1989b). Statistical method for testing the neutral mutation hypothesis by DNA polymorphism. Genetics 123: 585-595.

Team RDC (2013). R: a Language and Environment for Statistical Computing. R Foundation for Statistical Computing: Vienna, Austria.

Turner TL, Hahn MW, Nuzhdin SV (2005). Genomic Islands of Speciation in Anopheles gambiae. PLOS Biol 3: e285.

Wadsworth CB, Woods WA, Hahn DA, Dopman EB (2013). One phase of the dormancy developmental pathway is critical for the evolution of insect seasonality. J Evol Biol 26: 2359-2368.
Wanner KW, Nichols AS, Allen JE, Bunger PL, Garczynski SF, Linn CE et al. (2010). Sex pheromone receptor specificity in the European corn borer moth, Ostrinia nubilalis. PLOS ONE 5: e8685.

West J, Via S (2008). The genetic mosaic suggests a new role for hitchhiking in ecological speciation. Mol Ecol 17: 4334-4345.

Wickham H (2009). Ggplot2: Elegant Graphics for Data Analysis. Springer-Verlag New York Incorporated.

Wu C-I (2001). The genic view of the process of speciation. J Evol Biol 14: $851-865$.

Supplementary Information accompanies this paper on Heredity website (http://www.nature.com/hdy) 